\title{
PEDAGOGÍA DECOLONIAL Y EDUCACIÓN COMUNITARIA:UNA POSIBILIDAD ÉTICO-POLÍTICA
}

\begin{abstract}
Resumen
En el presente artículo se realiza una apuesta por la pedagogía decolonial como posibilidad de investigación de las prácticas de formación en la Educación Comunitaria, ECo, que se dan contemporáneamente. Se encuentra articulado en tres apartados, a saber: acercamientos históricos dados entre la Eco y la pedagogía en contextos universitarios, la formación ético-política en las prácticas de la Eco: una crítica a la epistemología del saber como poder y la pedagogía decolonial como una crítica al discurso pedagógico moderno. Las preguntas en torno a las cuales gira la discusión son entre otras: ¿a qué tipo de prácticas formativas hacemos referencia cuando hablamos de la Eco? ¿Cuál es el proyecto ético-político en el que se inscriben? ¿Cómo se da la producción de conocimiento en dichas prácticas de formación? ¿Desde dónde se legitima ese conocimiento? ¿Como se ubica la Eco en los actuales tiempos de capitalismo cognitivo y trabajo inmaterial en contextos de globalización? ¿Cómo se está asumiendo la crítica y desde dónde? ¿Cómo se articula el proyecto ético-político de la Eco al pensamiento crítico latinoamericano?
\end{abstract}

Palabras clave: Pedagogía, discursos decoloniales, formación ético-política, Educación comunitaria.

\section{PEDAGOGIA DECOLONIAL COMUNIDADE E EDUCAÇÃO: UMA POSSIBILIDADE ÉTICO-POLÍTICO}

\section{Resumo}

No presente artigo realiza-se uma aposta pela pedagogia decolonial como uma possibilidade de pesquisa das praticas de formação ético - políticas em Educação Comunitária -ECO- dadas na contemporaneidade. A aposta encontra-se articulada em três apartados: aproximações históricas entre a ECO e a pedagogia em contextos universitários, a formação ético - política nas praticas da ECO: uma crítica à epistemologia do saber como poder e a pedagogia decolonial como uma critica ao discurso pedagógico moderno. As perguntas ao redor das quais gira a discussão são entre outras: ¿A que tipos de práticas formativas referimo-nos quando se fala da ECO? ¿Qual é o projecto ético-político no que se inscrevem? ¿Como se dá a produção de conhecimento em ditas práticas de formação? ¿Desde onde se legitima esse conhecimento? ¿Como se localiza a ECO nos atuais tempos de capitalismo cognitivo e trabalho imaterial em contextos de globalização? ¿Como se assumi a critica e desde onde? ¿Como se articula o projeto ético-político da ECO ao pensamento crítico latino-americano?

Palavras chave: Pedagogia, discursos decoloniales, formação ético-política, Educação comunitária.

* Profesora de la Facultad de Educación, Universidad Pedagógica Nacional. Investigadora del grupo: Pedagogía y política. Correo electrónico: dianami29@gmail.com-dpenuela@pedagogica.edu.co

Articulo recibido el 22 de mayo de 2009 y aprobado el 14 de mayo de 2009 


\title{
DE-COLONIAL PEDAGOGY AND COMMUNITY EDUCATION: AN ETHICAL-POLITICAL POSSIBILITY
}

\begin{abstract}
In this article the de-colonial pedagogy is analyzed as a possibility of research of the teaching practices of ethical - political formation in Community Education (COE), given nowadays. It is articulated in three sections: historical approaches given between COE and the pedagogy in university contexts, the ethical - political formation in the practices of COE: a critique to the epistemology of the knowledge as power and the de-colonial pedagogy as a critique to the modern pedagogic speech. Some questions, which discussion mixes with, are: What type of formative practices do we refer to when we speak about COE? What is the ethical - political project in which they are registered? How is the production of knowledge given in the practices of formation above? Where is this knowledge recognized from? How is COE located in the current times of cognitive capitalism and immaterial work in contexts of globalization? How and where is being critique assumed from? How is the ethical - political project of COE articulated to the Latin-American critical thought?
\end{abstract}

Keys words: Pedagogy, de-colonial speeches, ethical - political formation, Community education.

En los actuales contextos de hegemonía de pensamiento, capitalismo cognitivo ${ }^{1}$, trabajo inmaterial ${ }^{2}$, fragmentación del vínculo social ${ }^{3} y$, en especial, de implantación de discursos que fácilmente pueden conducirnos al cinismo ético o intelectual, pues siguiendo a Ubilla "aparentemente sólo queda la posibilidad de aceptar la realidad tal cual es, aunque suponga marginación, empobrecimiento y exclusión" (Ubilla, 2004, p. 65), se hace necesaria la reconceptualización de las teorías y perspectivas críticas con las cuales se han estado leyendo las problemáticas de los contextos sociales. Varios autores ${ }^{4}$ han

1 Al respecto, ver: Mejía (2008). El autor señala que los procesos de reconfiguración mundial del capitalismo constituyen un nuevo proyecto de control como "el capitalismo cognitivo".

2 Hart y Negri (2001). Otros autores dicen que "el capital ha puesto a trabajar por cuenta propia la vida y sus manifestaciones". Al respecto, ver Blandeau (2004).

3 Aunque es de notar que autores como Pietro Barcellona (1999) hablan del regreso de la vinculación social, al respecto expone: "sostener la diferencia significa reivindicar una nueva determinación del espacio del encuentro, una nueva definición del espacio común... el único tiempo para una comunidad de diferentes es el tiempo de lo posible: el tiempo de la creación de un nuevo vínculo social".

4 Entre otros, Catherine Walsh, Walter

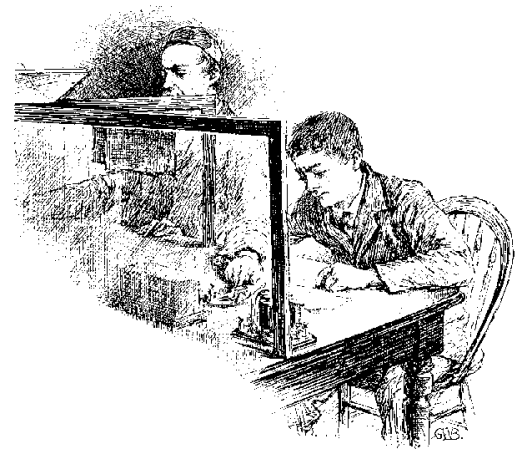

hablado de la necesidad de indisciplinar las ciencias sociales, mostrando la urgencia de descentrar sus locus de enunciación asépticos y alejados de la realidad, en busca de nuevas re-localizaciones a partir de las cuales escuchen, visualicen y construyan otros puntos de vista, otras realidades, desde un trabajo interdisciplinario.

Las posiciones academicistas neutrales y objetivas también son interrogadas desde el cuestionamiento de sus "ventanas epistemológicas", en el sentido de ir más allá de contemplar la realidad e intentar generar cambios sociales en contextos particulares y en los sujetos mismos. Es en este escenario donde se encuentra la práctica como el medio más cercano del que se dispone para abordar, reconocer y transformar sujetos y situaciones en contextos de profundas desigualdades sociales como los actuales.

Mignolo, Edgardo Lander y Santiago Castro.

\section{ALGUNOS ACERCAMIENTOS HISTÓRICOS DADOS ENTRE LA EDUCACIÓN COMUNITARIA Y LA PEDAGOGÍA EN CONTEXTOS UNIVERSITARIOS}

Ha sido preocupación constante de la Educación Comunitaria (Eco), establecer $y$, a la vez, problematizar sus relaciones con la pedagogía. En un primer momento, buscando distanciarse de su perspectiva funcionalista ${ }^{5}$ (Plan de Desarrollo Comunitario, 1992-1994), que emerge hacia la segunda mitad del siglo $\mathrm{XX}$, y en la cual expresiones como "desarrollo y educación comunitaria" se asociaron a los procesos de "integración" al nuevo orden de las poblaciones pauperizadas por efectos de la expansión capitalista, operando como uno de los medios más adecuados para controlar el conflicto social e incorporar-proceso

5 La cual en nuestro país se acentúa luego de la Constitución de 1991, en la que se fundamenta el nuevo contrato social entre los colombianos sobre claros principios de "participación ciudadana", como lo expresa el Ministro De la Calle Lombana hacia 1992: "La organización comunitaria ha llegado a la mayoría de edad; en lo futuro deberá ser autosuficiente. [...] En lo sucesivo exigirá sus derechos y el Estado deberá estar listo a responderle, pues en los planes de gobierno se desciende del concepto de participación democrática, al estadio más concreto de participación de la comunidad". 
que ahora se denomina inclusión por las políticas educativas- "participativamente" a los "marginales" a los procesos de modernización social.

En un segundo momento, la perspectiva de la ECO indaga por la pedagogía del trabajo comunitario, asumido tanto desde las llamadas prácticas comunitarias de las universidades en sus procesos de "extensión", dadas hacia la década del ochenta, como desde otras prácticas compartidas con la educación popular, como la educación de adultos y jóvenes EDJA, que en algunos lugares de Latinoamérica se denominó Escuela Popular Comunitaria de Adultos, Epca; experiencias educativas que pretendían introducir al sistema formal de educación de adultos algunos aspectos de la riqueza conceptual y metodológica acumulada por la educación popular (Magendzo, 1991, p. 7). De igual forma, se encuentran los desarrollos de quienes realizan educación en derechos humanos, de educación en salud, de género, con jóvenes, con indígenas, con desplazados por la violencia, con comunidades rurales o desde la teología que vienen creando elaboraciones conceptuales y trazando metodologías particulares apropiadas a sus campos temáticos.

Por otra parte, se han dado también procesos de teorización desde la academia que sitúan a la ECO como un campo educativo y un paradigma pedagógico emergente ${ }^{6}$ cuyos balances discursivos y prácticos están aún por realizarse, pero se convierten en un intento por acercar la universidad pública con las realidades sociales, buscando de manera concomitante combatir su crisis de legitimidad social ${ }^{7}$ desde el re-planteamiento de los programas ético-políticos y pedagógicos de la misma, más allá de lo escrito en el papel.

6 Al respecto ver: La Educación Comunitaria como campo educativo y pedagógico emergente, 1995. Documento de la Línea de Educación Comunitaria de la Maestría en Educación actualmente en reconstrucción.

7 Boaventura de Souza (2006) habla de tres tipos de crisis institucional que aquejan a la Universidad Pública, a saber: crisis de hegemonía, crisis de legitimidad y crisis institucional.
La Educación Comunitaria tiene la posibilidad de cuestionar el ethos universitario que se ha establecido históricamente al asumir como conocimiento formal y válido el conocimiento científico, invisibilizando la posibilidad del diálogo intercultural con otros tipos de saberes externos a la misma y que se producen en los microcontextos sociales, situación que es planteada por De Sousa como la posibilidad de hablar de conocimiento pluriuniversitario:

Como un conocimiento contextual que se produce extra-muros, transdisciplinario, que obliga a un diálogo con otros tipos de conocimiento, lo que lo hace internamente más heterogéneo y más adecuado a ser producido en sistemas abierto con carácter cooperativo y solidarios mediante movimientos sociales, organizaciones no gubernamentales, grupos sociales tales como inmigrantes, ancianos, comunidades populares y grupos de ciudadanos críticos y activos (Boaventura, 2006, pp. 38-39).

Esto en contravía de la tendencia política "de reducir la autonomía de la Universidad hasta el nivel necesario para eliminar la producción y divulgación libre del conocimiento crítico" (Boaventura, 2006, pp. 15-19).

Lo anterior permite cuestionar la relación dada entre la docencia y la extensión en nuestra Universidad desde las prácticas de formación tanto en pregrado como en posgrado en el marco de la Educación Comunitaria. Mediante las prácticas y salidas de campo, la Universidad ha tratado de "promover asociaciones activas en el campo pedagógico y científico con las escuelas públicas y las comunidades", no obstante, la concepción sigue siendo mirar desde adentro hacia afuera sus procesos de formación frente y no "con los otros," procesos externos de producción de saberes como expresión jerárquica de la posesión del "conocimiento científico". De igual forma, se cree que los procesos de discriminación "positiva" que funcionan para permitir el acceso de minorías étnicas (negritudes e indígenas), otorgando algunos cupos de acceso, determinan la función social de la Universidad y son equiparables a los diálogos interculturales que se deberían generar como parte del ethos universitario.
El siguiente apartado retoma estas preocupaciones y acercamientos históricos dados entre la ECO y la pedagogía en contextos universitarios, en la dirección de generar conexiones entre los discursos académicos contemporáneos sobre decoloniali$\mathrm{dad}^{8}$ que circulan en las universidades y las prácticas de formación que se desarrollan en diferentes escenarios educativos formales, no formales e informales.

\section{LA FORMACIÓN ÉTICO-POLIITICA EN LAS PRÁCTICAS DE LA ECO: UNA CRÍTICA A LA EPISTEMOLOGÍA DEL SABER COMO PODER}

Las prácticas de formación de la Eco son políticas, dado que las formas y modos de "leer" la pedagogía y la educación en contexto están vinculados de manera ineluctable con el poder, y son éticas desde la perspectiva de lectura de los sujetos, en el sentido en que se afirma una ética de la vida que se convierte luego en una crítica ética, en términos de Dussel:

Es decir, la vida humana es el contenido de la ética, desde donde se afirma la dignidad negada de la vida de la víctima, del oprimido o excluido. Las éticas críticas tienen como principio la obligación de producir y desarrollar la vida humana concreta de cada sujeto ético en comunidad 9 .

Pero no sólo en razón de la vida misma, sino en el sentido del deber ético de los sujetos (educando y educador) de intentar cambiarse primero a sí mismos y sus prácticas pedagógicas y luego a los contextos

8 La decolonialidad, como fundamentaremos más adelante, encuentra su razón desde los esfuerzos por situar lo propio y desde lógicas otras y pensamientos otros.

9 Dussel plantea que existen varios momentos de la ética a saber: El momento material de la ética que corresponde a la verdad práctica sobre qué es la ética de la vida, el momento de la moralidad formal o de la validez intersubjetiva, y el momento de la crítica ética, la validez antihegemónica de la comunidad de las víctimas y la praxis de la liberación (cursivas añadidas). 
y las situaciones que desde el campo de lo educativo contribuyen a perpetuar la realidad social como única y dada.

Ahora bien, si toda práctica educativa es política, siguiendo a Freire (2001), asimismo la práctica política es educativa. Las prácticas políticas siempre lo son porque involucran valores, proyectos y utopías que reproducen, legitiman, cuestionan o transforman las relaciones de poder prevalecientes en la sociedad; la educación nunca es neutral, está a favor de la dominación o de la emancipación. Por eso, Freire distingue entre prácticas educativas conservadoras y progresistas. ¿A que tipo de prácticas formativas hacemos referencia cuando hablamos de la ECO? ¿Cuál es el proyecto ético-político en el que se inscriben? $Y$ en este campo se multiplican los interrogantes más que las certezas ¿Cuáles son las posturas ético-políticas de los intelectuales y académicos cuyos discursos críticos buscan permear la escuela y los escenarios comunitarios? ¿Cómo se da la producción de conocimiento en dichas prácticas de formación? ¿Desde dónde se legitima ese conocimiento? ¿Cuáles son los procesos de historicidad de sus prácticas? ¿Se ha problematizado la relación entre communitas e inmunitas, entre lo común y lo propio, entre el sujeto y el colectivo? ¿Cómo se da su proceso de emergencia y configuración en el marco de los discursos de la modernidad y los procesos de modernización? ¿Cómo se ubica la ECO en los actuales tiempos de capitalismo cognitivo y trabajo inmaterial en contextos de globalización? ¿Cómo se está asumiendo la crítica y desde dónde? ¿Cómo se articula el proyecto ético-político de la Eco con el pensamiento crítico latinoamericano?

La pregunta por lo ético y lo político en las prácticas pedagógicas de formación no pretende dar respuesta a todos los cuestionamientos anteriormente formulados, pero sí pretende situar la práctica pedagógica desde una crítica a la epistemología del saber como poder; es decir, una crítica a la manera en que el poder es ejercido desde el punto en que tradicionalmente se ubica el conocimiento científico asumido como válido, aunque en muchos casos no legítimo, y en el cual, consecuentemente, quien sabe más, puede más. En el marco de lo esbozado, se considera pertinente ubicar la pregunta por la ética, pues es el elemento llamado a conducir la posibilidad de configurar nuevas relaciones sociales, en las cuales no sólo se consideren otras formas de entender el poder, sino, y de manera prioritaria, se dé efectivamente una redistribución diferente del poder. Así, la ética como componente de las relaciones pedagógicas, entendidas en el marco amplio de relaciones sociales particulares, posibilita mantener vivo el horizonte de posibilidad, siguiendo a Zemelman $^{10}$, y pensar otras formas de construcción del poder desde la emergencia de prácticas, constituidas por y, a la vez, constituyentes de sujetos, prácticas de libertad que problematizarían situaciones discursivas y prácticas que conllevan exclusiones, desigualdades, injusticias e invisibilización de las diferencias.

En este sentido, Mejía (2008) muestra la manera en que la teoría crítica, en sus diferentes vertientes euronorteamericanas, crea alianzas en la denuncia y propuesta frente al capitalismo occidental, pero considera que no es suficiente para explicar las particularidades de éste en nuestras realidades:

Por ello se necesita ser complementada con las especificidades de las relaciones sociales gestadas en el proceso de la colonialidad, que en el caso de los países de la periferia se ha dado como dominación, control y explotación no sólo en el ámbito del trabajo, sino en los ámbitos de la razón, el género, y los saberes relacionados a ellas, dando

10 Se trata de fijar un nuevo horizonte de posibilidad desde el cual pensar, antes que estructurar un nuevo discurso que remplace al anterior (Cfr. Zemelman, 1998).

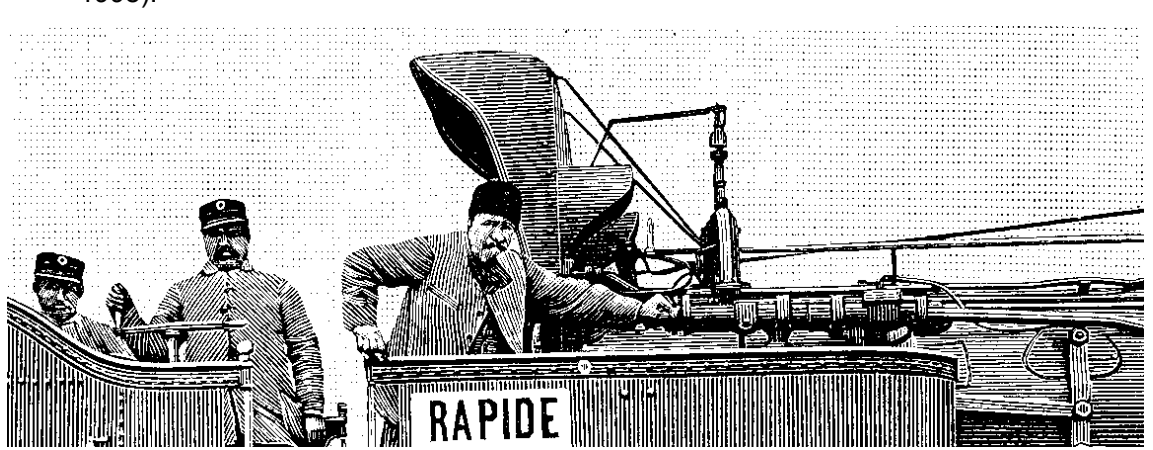

forma a estructuras que construyen una subjetividad controlada. Además, se ve la necesidad de incorporar una dimensión étnica y epistémica al conflicto, ya que opera en los sujetos pero como modos de conocer, de producir saber y conocimiento, de producir imágenes, símbolos, hábitos, significación y modos de relacionarse (Mejía, 2008) ${ }^{11}$.

En este punto, emerge el tercer referente conceptual que se aborda en la pregunta por la pedagogía decolonial y las relaciones de poder (hegemonía y dominación). Se parte de asumir que la función de la pedagogía:

No es la de ser reproductora de las relaciones de dominación y dependencia, por medio de las cuales se instaura un estilo educativo que no tiende a la formación de nuevos sujetos educadores y educandos, sino a su dependencia y subordinación (Ubilla, 2004, p. 71).

Es decir, no hablamos de una pedagogía funcional, sino de una pedagogía de la resistencia. Aquí se consideran funda-

11 El autor en mención propone retomar las vertientes de crítica en contextos latinoamericanos, como en la teoría de la dependencia, la teología de la liberación, la psicología social latinoamericana, en la educación popular, la filosofía latinoamericana, el teatro del oprimido, los teóricos de la colonialidad, la comunicación popular y las formas críticas del desarrollo a escala humana, entre otras.

\section{LA PEDAGOGÍA DECOLONIAL: UNA POSIBILIDAD CRÍTICA AL DISCURSO PEDAGÓGICO MODERNO}


mentales los aportes freirianos en su comprensión, los cuales han sido apropiados y resignificados en distintos escenarios, en procesos de resolución de conflictos, desde una perspectiva pedagógica, en procesos de construcción de proyectos pedagógicos y educativos alternativos orientados por los principios de la Educación Popular, en procesos de educación intercultural, en movilizaciones pedagógicas como el denominado Movimiento Pedagógico $^{12}$ de la década del ochenta, y desde las prácticas, que no así desde los discursos, en procesos como la Expedición Pedagógica Nacional con sus inconsistencias institucionales y las Redes de maestros con sus alcances y limitaciones, entre otros.

Aunque existen varias corrientes que en nuestro país han situado y posicionado la pedagogía desde lugares distintos, en ocasiones contradictorios entre sí, se asume la corriente pedagógica freiriana y sus posteriores desarrollos y apropiaciones ${ }^{13}$ desde sus acercamientos con la educación popular y la educación comunitaria, dado que es una pedagogía contextual que nace desde y para América Latina, se asume la posibilidad de generar diálogos con posturas y corrientes históricas eurocéntricas ${ }^{14}$. Así pues una de las preguntas que mueve el presente artículo desde los discursos decoloniales, es indagar

12 Al respecto, ver la tesis de maestría, escrita en coautoría con el profesor Víctor M. Rodríguez, titulada: El Movimiento Pedagógico: un espacio para la resistencia y el ejercicio de poder. [en prensa]. Concurso Francisca Radke.

13 Sitúo, entre otros, a José Luis Rebellato con su praxis dialógica comunitaria, Moacir Gadotti, Claudia Korol, Óscar Jara desde la sistematización a la renovación teórico-práctica de los movimientos sociales, entre otros.

14 Las aplicadas indagaciones históricas al respecto se han dado en nuestro país por medio de las investigaciones del Grupo de Historia de las práctica pedagógicas, GHPP, trabajando, entre otros: Herbart, Pestolozzi, Comenio, Rosseau, así como las apropiaciones de la Escuela Activa, que se dieron en varias experiencias de Educación Popular. si desde las prácticas, no desde los discursos, se puede hablar de pedagogías otras desde América Latina y por medio de las prácticas y procesos realizados en el marco de la Educación Popular y la Educación Comunitaria, ¿Se estará hablando sólo de procesos de apropiación de escuelas y discursos eurocéntricos? O ¿quizás existe la posibilidad que se hayan constituido una o varias pedagogías a partir de híbridos conceptuales y prácticos en algunos casos, pero también saberes propios pedagógicos en América Latina y, específicamente, en Colombia?

La pedagogía decolonial en el presente artículo, se asume desde la crítica que Cullen (2004) le realiza al discurso pedagógico moderno ${ }^{15}$, pues se considera necesario que la pedagogía de las prácticas de formación comunitaria y los discursos de la Educación Comunitaria hagan explícito su lugar de ubicación histórica, tanto en los discursos del pensamiento crítico latinoamericano como en las problematizaciones frente a los discursos de la modernidad y los procesos de modernización.

De manera preliminar, se puede decir que la lectura crítica de la realidad propia de la ECO y compartida con la Educación Popular, EP, se puede plantear desde una crítica que cuestione no sólo el contexto de política pública internacional, nacional y local, sino que problematice en sí misma la colonialidad del saber (Mignolo, 2005) y las geopolíticas de distribución del conocimiento, a partir de reconocer la emergencia de nuevas perspectivas que cuestionan los metarelatos, las visiones totalizantes y eurocéntricas y que avanzan en la crítica al estatuto de las disciplinas, entre otros aspectos. En este sentido, se considera importante la visibilización de esas otras formas de relación entre saber y conocimiento, entre sociedad y cultura

15 Entre otras críticas, plantea: la configuración del discurso del método, contrato o mercado; la articulación del discurso pedagógico moderno en una trama de prácticas educativas controladas, desde una lucha por la hegemonía y la articulación con prácticas educativas fragmentadas desde una tensión por la competitividad que se expresa en la división social de los saberes enseñados, entre otros aspectos. y el análisis in situ de las relaciones de poder en la distribución, legitimidad y jerarquización del mismo. Fundamentados en la comprensión de esto, se aportaría discursivamente en el posicionamiento de la ECO en el campo del pensamiento crítico latinoamericano. A continuación, se presenta de manera breve algunos aportes de los discursos decoloniales en el campo de lo pedagógico.

En los discursos decoloniales, el proyecto de modernidad-colonialidad ha sido discutido y problematizado desde diferentes perspectivas por múltiples autores, entre otros, Mignolo, Walsh, Coronil, Escobar, Dussel $^{16}$, Lander ${ }^{17}$, Quijano, MaldonadoTorres y Castro. Aunque sus formas de asumir y entender el poder son diferentes, especialmente desde Castro y Escobar, podríamos decir que tienen en común aceptar que el proyecto de la modernidad-colonialidad introdujo un cambio en la organización tiempo-espacio de las culturas, pueblos y territorios del planeta, presentes y pasados, tratando de vincularlos en una gran narrativa universal. Así, hacen referencia a poder colonial, poder imperial, como moduladores de una universalidad excluyente, de una historia de invisibilizaciones discursivas y reales simultáneamente y como generador de estrategias geopolíticas de producción del conocimiento con la consecuente invisibilización de otras territorialidades discursivas. Se comprende entonces la necesidad por parte de las prácticas de formación de la ECO de asumir la crítica desde la posibilidad de descolonizar nuestra propia historia para vernos ante el espejo de nuestras realidades propias.

16 Dussel (1989, p. 47) sitúa a América Latina como la otra cara de la modernidad. Dice que América Latina nació al mismo tiempo que la "modernidad", pero como su otra cara, necesaria, silenciada, explotada y dominada.

17 Dice el autor que, además, este metarrelato de la modernidad es un dispositivo de conocimiento colonial e imperial Así pues, Las otras formas de ser, las otras formas de organización de la sociedad. las otras formas del saber son trasformadas no sólo en diferentes, sino en carentes, en arcaicas, primitivas, tradicionales, premodernas. 
No se trata de invisibilizar al otro (en este caso, los discursos eurocéntricos), pero sí de generar posturas dialógicas, retomando a Freire, e interculturales a partir de situarnos de manera práctica y discursiva desde lo que somos y hacemos.

De igual manera, se incluye aquí tanto la noción de pensamiento de frontera, planteada por Mignolo, como el posicionamiento crítico de frontera, que expone Walsh $^{18}$. El primero se retoma a partir de la necesidad de la pedagogía decolonial de generar posibilidades de pensamientos otros que permitan fronterizar el pensamiento hegemónico, a manera de proyectos contra-hegemónicos, para posteriormente permitir que los sujetos se posicionen críticamente, es decir, transgredan el límite del pensar al actuar. Retomamos la noción de hegemonía de Gramsci, pues "Si la hegemonía es ético-política, no puede dejar de ser también económica" (Acanda, 2007, p. 241). Sin embargo, es evidente que, de manera paralela a esta hegemonía económica, coexiste una hegemonía cultural: "Cuando hablamos de hegemonía, estamos haciendo referencia a proceso históricos, a formas de vida y de autorrealización de los seres humanos individuales" (Gramsci, 2005, p. 173).

Introduce así Gramsci el problema del sujeto en relación con la fuerza hegemónica, pues en la base de su teoría subyace una

18 Explorando la categoría de "posicionamiento crítico fronterizo" que, en contraposición al pensamiento fronterizo de Mignolo (producto desde la subalternidad y desde las fronteras o intersticios de los lógicas de pensamiento -la dominante y la subalterna- de hacer visibles otras lógicas y maneras de pensar diferentes a la eurocéntrica y dominante, poniendo a ellas en diálogo o disputa con la dominante), el posicionamiento crítico fronterizo rescata el pensamiento otro (noción de Abdelkebir Khatibi, pensador árabe islámico), el que se construye casa adentro, el que permite hacer resaltar la agencialidad de los grupos subalternizados no sólo para incidir en o para fronterizar el pensamiento hegemónico, sino también para moverse estratégicamente en una variedad de esferas". Su meta es la interculturización del pensamiento (Walsh, 2005, p. 17). comprensión relacional del poder, según Acanda, en contra de la concepción liberal de poder, en la cual éste es concebido como un bien que se posee $\mathrm{y}$, mediante acuerdo, se cede, así:

La hegemonía de una clase es manifestación de su capacidad para encontrar formas nuevas de manejar los conflictos sociales, de cooptar y quitarle su filo subversivo a las nuevas manifestaciones de resistencia surgidas desde otras clases sociales, de recomponer constantemente los equilibrios perdidos. La hegemonía nunca es inmóvil, es la expresión de la lucha de clases, de las "relaciones de fuerza" dinámicas que constantemente se renuevan en una sociedad (Acanda, 2007, p. 173).

La hegemonía se puede expresar tanto en la dependencia externa (por ejemplo, los procesos de cooperación económica internacional) como en las relaciones sociales de dominación, denominado por Boaventura de Sousa como fascismo societario $^{19}$, que emerge como una expresión de dichas relaciones sociales de dominación, las cuales pasan por la aceptación y apropiación acrítica de los discursos hegemónicos por parte de los sujetos.

Ahora bien, si se retoma lo enunciado hasta ahora, y añadiendo otros elementos, se sitúan los posibles ejes conceptuales de la pedagogía decolonial así:

\section{LA CRÍTICA}

Partir de un proyecto de crítica a las razones de educar (¿Para qué se educa? ¿Por qué se educa?) como una posibilidad de formación que permita que los sujetos involucrados en la constitución de saberes y de conocimiento se piensen en primer lugar a sí mismos desde lo que hacen, a par-

19 El fascismo societario está formado por una serie de procesos sociales mediante los cuales grandes segmentos de la población son expulsados o mantenidos irreversiblemente fuera de cualquier tipo de contrato social. En tanto régimen societario, el fascismo se manifiesta como el colapso de las más triviales expectativas de la gente que vive bajo su dominio. tir de la reflexión sobre su práctica, desde su historicidad como sujetos (sus historias de vida), elemento compartido tanto por la educación popular como la comunitaria. El papel de la memoria "peligrosa". Y, por supuesto, la crítica situada también en el funcionamiento de las políticas educativas en sus diferentes niveles (internacional, nacional, distrital y local).

\section{LA PEDAGOGÍA DE LA PREGUNTA Y SU VALIDEZ COMO CONDICIÓN DE LA EXISTENCIA HUMANA}

Esto, asumiendo la importancia de la pedagogía de la pregunta como generadora de un pensamiento desafiante, que antes contextualizamos como pensamiento de frontera, a partir de la reflexión sobre la práctica en la formulación de proyectos y propuestas de investigación, buscando que educadores y educandos piensen y actúen sobre sus realidades por medio de la identificación de los problemas sociales, culturales y políticos que pueden ser intervenidos de manera propositiva desde la educación, elemento también retomado desde la ECO y la Educación Popular.

\section{LAS RELACIONES DE PODER O EL PODER EN EJERCICIO}

Analizando el funcionamiento ${ }^{20}$ de las relaciones de poder (proyectos hegemónicos, relaciones de dominación, dependencia, represión) a partir de contextos particulares específicos (procesos de formación inicial y de posgrado en el campo de la educación comunitaria, funcionamiento de organizaciones comunitarias, ONG, centros alternativos de prácticas alternativas de comunicación, entre otros), análisis de las prácticas de formación en la política educativa (por ejemplo, los comedores comunitarios, Programa Escuela-CiudadEscuela, programas de cultura ciudadana) y análisis de proyectos rurales comunitarios. De manera metodológica, la noción

20 Se asumen elementos de deconstrucción en el marco de un posmodernismo oposicional, como propone Boaventura de Souza. 
de dispositivo ${ }^{21}$ podría ser útil, pero, a partir de situar en primer lugar históricamente el proceso, movilización o proyecto de educación comunitaria, ¿quiénes hablan? ¿Desde dónde lo hacen? ¿Qué prácticas, saberes y discursos agencian? ¿Cuál es el proyecto ético-político que promueven? ¿Cuáles son los niveles de relación de su proyecto alternativo o en búsqueda de una nueva institucionalidad con la política internacional, nacional, distrital y local? Esta es una opción por la duda, pero no una duda que inactive, sino una duda que nos des-localice de nuestras seguridades no reflexionadas, que cree en los sujetos (tanto educandos como educadores) la necesidad de decidir, optar, actuar, como veremos más adelante.

\section{LA FORMACIÓN ÉTICO-POLÍTICA $Y$ LOS HORIZONTES DE POSIBILIDAD}

Por medio de la identificación de tensiones y posibilidades de los procesos de formación ético-política, la pedagogía decolonial se ocupa también de hacer explícitas, en los procesos de formación, las discusiones sobre categorías como la ética, las formas de lo político, los valores de mercado (autoritarismo, tecnocracia, utilitarismo, individualismo) y los valores de vínculo social (comunicación dialógica, justicia social, dignidad humana), así como la pregunta por el sujeto, para lograr hacer evidente que las relaciones de dominación y dependencia externa pueden condicionar y no determinar, en la medida en que tanto educadores como educandos logremos identificar los procesos de autodominación y control que de manera acrítica ejercemos sobre nosotros mismos, nuestras prácticas y comprensiones de la realidad $^{22}$. La idea es que esta pedagogía

21 "Un dispositivo es un conjunto resueltamente heterogéneo, que incluye, discursos, instituciones, instalaciones arquitectónicas, decisiones reglamentarias, leyes, medidas administrativas, [....] lo dicho y también lo no dicho, estos son los elementos del dispositivo. El dispositivo mismo es la red que se establece entre estos elementos" (Foucault, 1991).

22 Siguiendo a Foucault, una mirada a decolonial enriquezca los valiosos aportes de la ECO, pero desde una postura que sospeche y posibilite que, si la realidad es construida por los sujetos, existe un marco de posibilidad de cambio de las situaciones de injusticia, conflictividad y exclusión que efectivamente vivimos en estos tiempos de globalización. Dado que lo decolonial representa una estrategia que va más allá de la transformación (lo que implica dejar de ser colonizado) apuntando mucho más que a la transformación, a la construcción o a la creación. Y si antes ubicamos la pregunta como generadora de pensamiento desafiante, la pregunta por lo ético permite que los sujetos asumamos, a partir de ese pensamiento de frontera, posiciones y posturas crítico-éticas, o lo que denominamos en los referentes conceptuales como posicionamiento crítico de frontera, es decir, las acciones concretas pero reflexionadas.

\section{INTERDISCIPLINARIEDAD 23 Y USO DE METODOLOGÍAS CUALITATIVAS CRÍTICAS}

Asumir la importancia de plantear y generar proyectos e investigaciones de manera interdisciplinaria, por medio del uso de metodologías cualitativas críticas como narrativas, reconstrucción de la memoria colectiva, historia oral, la sistematización tanto de corriente hermenéutica como antropológica, etnografía y teoría de la observación propuesta Juan Manuel Delgado, talleres de investigación, estudios de caso, asumiendo la vigencia que éstos tienen en su pre-eminencia a los sujetos, sus narraciones, historias de vida, subjetividades, sentimientos, deseos, voluntades, expresiones políticas y acciones colectivas.

nuestros microfascismos, tesis que expone en Microfísica del poder.

23 Generando diálogos con la historia social, la antropología simbólica, la comunicación social, la sociología de la cultura, las teorías de la recepción cultural, la psicología social y educativa, algunas vertientes del constructivismo con énfasis en lo cultural, el análisis institucional, entre otros.

\section{CONSTRUCCIÓN DE CONTRA-HEGEMONÍA}

Esto mediante la organización, articulación y sostenibilidad de los proyectos de formación ético-política que se inscriben dentro de la Educación Comunitaria.

\section{PARA ABRIR LA DISCUSIÓN}

La pedagogía decolonial emerge como una posibilidad investigativa para problematizar, de-construir y re-construir las prácticas de formación ético-políticas, en el marco del capitalismo de la información y la comunicación, así como la sociedad de consumo, implícitas y explícitas en los diferentes proyectos y procesos de la ECO, al igual que en los proyectos sociales hegemónicos (formación de qué tipo de ciudadanía y pre-eminencia de qué tipo de valores, como se esbozo anteriormente), tanto en escenarios de educación formal, no formal como informal. Su pretensión es pues realizar de forma paralela, pero con un espectro de mayor duración en el tiempo, un proceso de indagación sobre la descolonización de la pedagogía como discurso y práctica en los procesos de colectivización y generación de vínculos comunitarios, asumiendo la posibilidad de hablar de pedagogía de la resistencia, a partir de la visibilización y caracterización de pedagogías otras, en las cuales surgen cuestionamientos ya enunciados en el escrito, como ¿Se puede hablar de pedagogías otras desde América Latina y mediante las prácticas y procesos realizados en el marco de la Educación Popular y la Educación Comunitaria, o se estará hablando sólo de procesos de apropiación de escuelas y discursos eurocéntricos? 0 quizás ¿Se han constituido pedagogías a partir de híbridos conceptuales y prácticos de discursos eurocéntricos en algunos casos, pero también de saberes propios pedagógicos en América Latina y específicamente en Colombia?

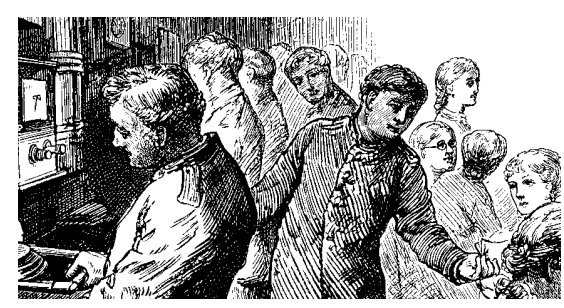




\section{BIBLIOGRAFÍA}

AcAndA, J. (2007). Traducir a Gramsci. La Habana: Editorial de Ciencias Sociales.

Agamben, G. (1996). La comunidad que viene. España: Re-textos.

Barcellona, P. (1999). Posmodernidad y comunidad. El regreso de la vinculación social. Madrid.

BlandeAu, O. (2004). Capitalismo cognitivo, propiedad intelectual y creación colectiva. Madrid: Traficante de Sueños.

BoAventuRA DE S. (octubre-diciembre, 2004). Nuestra América: reinventando un paradigma. (Fragmentos). Casa de las Américas [La Habana], año XLV, núm. 237, pp. 7-25.

(2006). La universidad en el siglo XXI. Para una reforma democrática y emancipadora de la Universidad. Casa de las Américas.

CASTRO, S. (2007). Michel Foucault y la colonialidad del poder. Revista Tabula Rasa, 6 , pp. 153-172.

Cullen, C. (2004). Perfiles ético-políticos de la educación. Buenos Aires: Paidos.

Deleuze, G. y Guattarl, F. (2005). ¿Qué es la filosofía? Barcelona: Anagrama.

Esposito, R. (2003). Communitas. Origen y destino de la comunidad. Argentina: Amorrortu Editores.

Documento Línea Maestría en Educación COMUNITARIA (en proceso de reconceptualización). (1995). Departamento de posgrado Maestría en Educación. Universidad Pedagógica Nacional.

Dussel, E. (1989). Fundamentos de la ética y la filosofía de la liberación. Madrid: Siglo XXI Editores.

Dussel, E. (1998). Ética de la liberación en la edad de la globalización y de la exclusión. Madrid: Trotta.

Dussel, E. (2007). La filosofía de la liberación ante el debate de la posmodernidad y los estudios latinoamericanos. En L. Tovar (ed.).
La posmodernidad a debate. Biblioteca Colombiana de Filosofía. Bogotá: Universidad Santo Tomás, USTA.

FouCAULt, M. (1991). Sabery verdad. España: La Piqueta.

(1979). Foucault, M. Microfísica del poder. España: La Piqueta.

Freire, P. (1980). Pedagogía del oprimido. Buenos Aires: Siglo XXI Editores.

(1993). Pedagogía de la esperanza. México: Siglo XXI Editores.

. (2001). Política yeducación. Siglo XXI

Editores. México.

(2006). Pedagogía de la autonomía Saberes necesarios para la práctica educativa. México: Siglo XXI Editores.

Gramsci, A. (1976). Introducción a la filosofía de la praxis. Ediciones Península. Barcelona.

HARDt, M. y NEGRI, T. (2001). Imperio. Bogotá. Ediciones Desde Abajo.

La EduCACIÓN COMUNITARIA COMO CAMPO EDUCATIVO Y PEDAGÓGICO EMERGENTE. (1995). Documento en línea Maestría en Educación Comunitaria (en proceso de reconceptualización). Universidad Pedagógica Nacional.

LANDER, E. (2000). Ciencias sociales: saberes coloniales y eurocéntricos. En E. Lander (comp.), La colonialidad del saber: eurocentrismo y ciencias sociales. Perspectivas latinoamericanas. Buenos Aires: Clacso.

Magendzo, A., Mena, F. y Valdivia, L. (1991). Escuela Popular Comunitaria de Adultos. Santiago de Chile.

MEJíA, M. (2008). Las pedagogías críticas en tiempos de capitalismo cognitivo. Cartografiando las resistencias en educación. Ponencia. Maestros Gestores, Pedagogías Críticas y Resistencias, Medellín, 22 al 24 de mayo.

Mignolo, W. (2005). Cambiando las éticas y políticas del conocimiento. Tabula Rasa, 5. pp. 47-72.
Peñuela, D. y Rodriguez, V. El Movimiento Pedagógico: un espacio para la resistencia y el ejercicio de poder. En prensa. Concurso Francisca Radke, Universidad Pedagógica Nacional 2008-2009.

(2006). Movimiento Pedagógico: otras formas de resistencia educativa. Folios, 23, pp. 50-56.

Plan de Desarrollo Comunitario 1992-1994. Colombia. Ministerio de Gobierno.

Posada, J. (1993). Epistemología y sistematización en la Educación Popular y la Educación Comunitaria. Pedagogía y Saberes, 4, pp 7-13.

Rebellato, J. (2004). Paulo Freire: educación y proyecto ético-político de transformación. En Pedagogía de la Resistencia. Cuadernos de Educación Popular. Buenos Aires: Ediciones Madres de la Plaza de Mayo.

SENNET, R. (2003). El respeto. Sobre la dignidad del hombre en un mundo de desigualdad. Barcelona: Anagrama.

Torres. A. (2007). La Educación Popular. Trayectoria y actualidad. Bogotá EI Búho.

TORRES A. y JiMÉnEZ, A. (comp.). (2006). La práctica investigativa en ciencias sociales. Bogotá: Universidad Pedagógica Nacional.

UвเLA, P. (2004). Ética y pedagogía o recreando a José Luis Rebellato. En Pedagogía de la Resistencia. Cuadernos de Educación Popular. Buenos Aires: Ediciones Madres de la Plaza de Mayo.

WALSH, C. (2005). (Re) pensamiento crítico y (de) colonialidad. En Pensamiento crítico y matriz (de)colonial reflexiones latinoamericanas. Quito: EdicionesAbya-Yala. Universidad Andina Simón Bolívar.

ZemeLMAN, H. (1998). La razón como construcción: un repaso histórico de planteamientos. En Sujeto: existencia y potencia. México: Anthropos.

Zuluaga, O. et al. (2003). Pedagogía y epistemología. Bogotá: Magisterio. 